\title{
High-resolution In Situ imaging of Biological Samples with Warp and M
}

\author{
Dimitry Tegunov
}

Max Planck Institute for Biophysical Chemistry, Goettingen, Niedersachsen, Germany

In situ structural biology aims to capture the shape, interactions and organization of macromolecules inside their native cellular environment. Cryo-electron tomography, coupled with thin samples or advanced sample preparation techniques [1], offers an unparalleled level of detail for in situ studies [2, 3]. Instead of a single 2D projection, a series of images at different sample tilt angles (tilt series) is acquired. Once aligned, tomograms can be reconstructed from the tilt series to resolve the macromolecular organization in 3D. Tilt series data can also be subjected to single-particle analysis (SPA) to achieve even higher resolution by averaging the signal of multiple identical particles [4]. Unfortunately, the resolution of SPA maps obtained from in situ data has trailed far behind the results achievable for isolated molecules in vitro [5]. On the one hand, the crowded cellular environment and the relatively high sample thickness limit the available signal for each particle. On the other hand, accurate alignment of tomographic tilt series data poses a harder task than the 2D movies commonly acquired for in vitro samples, and is not yet handled optimally by the less mature tilt series software ecosystem [6].

$\mathrm{M}$ is a new tool that integrates with Warp [7] and RELION [8], and improves tilt series alignments by modeling the contents of each tomogram as a multi-particle system with hyperparameters describing its various modes of deformation. Individual particle poses are affected by the deformation model as a function of particle position, tilt angle and electron dose. The spatial and temporal coarseness of the model regularizes the solution and prevents the overfitting of physically implausible changes, as suggested previously [9]. M fits particle poses and deformation hyperparameters simultaneously by maximizing the correlation between projections of high-resolution maps and experimental image data using a gradient descent optimization. Coupled with advances in high-defocus contrast transfer function correction and 3D map denoising, $\mathrm{M}$ outperforms other implementations of reference-based tilt series refinement [10, 11]. Most notably, $\mathrm{M}$ is able to refine in situ data of $70 \mathrm{~S}$ ribosomes inside a $150 \mathrm{~nm}$ thick specimen to $3.7 \AA$, rivaling the resolution of comparable in vitro studies for the first time.

\section{References}

1. Schaffer, M., et al., A cryo-FIB lift-out technique enables molecular-resolution cryo-ET within native Caenorhabditis elegans tissue. Nat Methods, 2019. 16(8): p. 757-762.

2. Mahamid, J., et al., Visualizing the molecular sociology at the HeLa cell nuclear periphery. Science, 2016. 351(6276): p. 969-72.

3. Engel, B.D., et al., Native architecture of the Chlamydomonas chloroplast revealed by in situ cryoelectron tomography. 2015.

4. Guo, Q., et al., In Situ Structure of Neuronal C9orf72 Poly-GA Aggregates Reveals Proteasome Recruitment. Cell, 2018. 172(4): p. 696-705.e12.

5. Dong, Y., et al., Cryo-EM structures and dynamics of substrate-engaged human $26 S$ proteasome. Nature, 2019. 565(7737): p. 49-55.

6. Leigh, K.E., et al., Subtomogram averaging from cryo-electron tomograms. Methods Cell Biol, 2019. 152: p. 217-259.

7. Tegunov, D. and P. Cramer, Real-time cryo-electron microscopy data preprocessing with Warp. Nat Methods, 2019. 16(11): p. 1146-1152. 
8. Scheres, S.H., RELION: implementation of a Bayesian approach to cryo-EM structure determination. J Struct Biol, 2012. 180(3): p. 519-30.

9. Bartesaghi, A., et al., Protein Secondary Structure Determination by Constrained Single-Particle CryoElectron Tomography. Structure, 2012. 20(12): p. 2003-13.

10. Himes, B.A. and P. Zhang, emClarity: software for high-resolution cryo-electron tomography and subtomogram averaging. Nat Methods, 2018. 15(11): p. 955-961.

11. Chen, M., et al., A complete data processing workflow for cryo-ET and subtomogram averaging. Nat Methods, 2019. 16(11): p. 1161-1168. 